\title{
The Radiobiology of Conventional Radiotherapy and Its Application to Radionuclide Therapy
}

\author{
Roger Dale ${ }^{1,2}$ and Alejandro Carabe-Fernandez ${ }^{2}$ \\ ${ }^{1}$ Radiation Physics and Radiobiology, Charing Cross Hospital, London, UK \\ ${ }^{2}$ Faculty of Medicine, Imperial College, University of London, London, UK
}

\begin{abstract}
The linear-quadratic (LQ) model of radiobiological effect is well established in conventional, i.e., external beam, radiotherapy. Because the model is derived from sound biophysical principles, it is also emerging as the standard formalism for assessing biological responses for the whole range of radiotherapy treatments. A central feature of $L Q$ methodology is the quantity known as the biologically effective dose $(B E D)$, which may be used to quantify the radiobiological impact of a treatment on both tumors and normal tissues. The BEDs commonly associated with conventional therapy may thus be compared to those expected from novel radiotherapy treatments, such as targeted radionuclide therapy. This approach also provides a mechanism for designing targeted treatments which are therapeutically equivalent to external beam treatments. In this paper the LQ methodology is outlined and worked examples are provided which demonstrate the tentative link between targeted radiotherapy doses and those used in conventional radiotherapy. The incorporation of an allowance for relative biological effectiveness (RBE) effects is also discussed. The complexity of the subject and the potentially large number of variables does place a restriction on overall predictive accuracy and the necessary caveats are outlined.
\end{abstract}

Key words: radiobiology, radionuclide therapy, targeted therapy, linear-quadratic model

\section{INTRODUCTION}

The linear quadratic (LQ) model of radiation effect has been discussed in a recent article in this journal and, thus, requires only an outline description here. ${ }^{1}$ The model provides a satisfactory explanation of fractionation and dose-rate effects observed at the macroscopic (clinical) level and may be applied to both tumor and normal tissues. ${ }^{2-4}$ It is, therefore, of particular use in evaluating how altered patterns of radiation delivery might impact on overall therapeutic index, that is

Address reprint requests to: Roger Dale; Radiation Physics and Radiobiology, Charing Cross Hospital; London W6 $8 R F$, United Kingdom

E-mail: r.dale@imperial.ac.uk to say, on the balance between the probabilities of tumor cure and normal tissue complications. As the LQ model is widely used in the analysis of more conventional radiotherapy, it may be used as a fulcrum for intercomparisons with more novel treatments, such as targeted radionuclide therapy.

\section{Types of Radiation Damage}

It is conventionally assumed that radiation damage occurs within the DNA, and two types of damage-formation process, both of which lead to cell lethality, may be postulated. Type A damage occurs when adjacent DNA strands are disrupted (broken) as a result of a single ionizing event. Type B damage results from an interaction between two proximal single-strand breaks created 
in separate ionizing events. The individual single-brand breaks (i.e., the precursors to Type B damage) are assumed to be potentially repairable and, hence, sublethal. It thus follows that if the damage from the first sublethal event repairs before the second event occurs, there will be no lethality.

Type A and Type B damage characteristics are summarized below. Sublethal, single-target damage is the key to understanding fractionation and dose-rate effects because, unlike the case with Type A damage, the amount of Type B damage is reduced when there are opportunities for sublethal repair, as occurs when a treatment is protracted.

\begin{tabular}{lc} 
Type A damage & \multicolumn{1}{c}{ Type B damage } \\
\hline $\begin{array}{l}\text { Always lethal. } \\
\text { Amount of damage is } \\
\text { always proportional } \\
\text { to dose. }\end{array}$ & $\begin{array}{c}\text { Always lethal. } \\
\text { For instantaneous exposures, } \\
\text { the amount of damage is } \\
\text { proportional to dose. }\end{array}$ \\
$\begin{array}{c}\text { Amount of damage is } \\
\text { independent of dose } \\
\text { rate and exposure } \\
\text { time. }\end{array}$ & $\begin{array}{l}\text { additionally dependent on } \\
\text { dose-rate and exposure time. }\end{array}$ \\
& $\begin{array}{l}\text { For any given dose, as } \\
\text { exposure time increases, the } \\
\text { amount of Type B damage is } \\
\text { reduced. }\end{array}$
\end{tabular}

\section{External Beam (XTB) Radiotherapy}

All radical external beam (XTB) radiotherapy is fractionated and usually entails the delivery of dose fractions of short duration (typically 1 minute or less) at intervals of approximately 24 hour. The individual dose fractions create both lethal (irreparable) and sublethal (repairable) damage. Because the sublethal damage (SLD) repairs with a half-life which is typically on the order of 0.5-3 hours, almost all of it is able to repair between fractions. Thus, although each fractional dose delivery reduces the cell population, those cells which are not sterilized, but which are sublethally damaged, recover themselves and respond to subsequent doses as if they had not previously been irradiated. There is thus no buildup of sublethal injury from earlier in the schedule and which could have been compounded to create extra-lethal damage. The radiosensitivity of the unsterilized cells remains the same at the time of each dose delivery. (It should be noted that, if the fractions are closely spaced, the gradual buildup of SLD allows for relatively more lethal damage to be created in each suc- cessive fraction, that is to say radiosensitivity effectively increases as treatment progresses.)

The interfraction SLD repair phenomenon allows tissues to be "spared" as a result of being subjected to fractionated radiation, i.e., for any given dose, the net cell kill is lower when the dose is fractionated than if it were all delivered in one single dose. As has been explained elsewhere, normal tissues generally have a greater capacity to repair SLD than tumors, and this provides the radiobiological rationale for conducting fractionated therapy. ${ }^{1}$ Provided the total dose is such that a good degree of tumor cure can be achieved, fractionation provides a degree of preferential "sparing" of the critical normal tissues and limits the morbidity. ${ }^{5}$

\section{Continuous Low-Dose-Rate (CLDR) Radiotherapy}

Continuous low-dose-rate (CLDR) radiotherapy normally extends over time periods of hours or days. As with XTB therapy, the continuous delivery of radiation creates both lethal (fixed) and sublethal (repairable) damage. However, because CLDR therapy is protracted, and because the rate of dose delivery is relatively low, some of the SLD has an opportunity to repair itself while the radiation is being delivered. The SLD repair therefore takes place as treatment progresses. Thus, as with XTB therapy, CLDR spares the normal tissues relatively more than the tumor, and the use of this modality confers, in principle, the same benefits as fractionation.

The radiobiological processes governing XTB and CLDR are thus seen to be essentially identical. They may be incorporated within the LQ model to allow for quantitative intercomparison of the two modalities.

\section{Targeted Radiotherapy (TRT)}

Targeted radiotherapy (TRT) is a form of continuous radiation delivery during which the doserate is not held constant but rises from zero to some maximum value and then drops back to zero. As with XTB and CLDR, the delivery of radiation at varying dose-rates creates both lethal and sublethal damage. Because TRT radiation delivery normally takes hours or days, some of the SLD repairs while the radiation is being delivered, but the differential between creating new damage and the repair of existing sublethal damage will now also depend on the dose-rate being delivered at any particular time. When tumors 
and critical normal tissues are each subjected to the same dose-rate profile, the sparing argument is similar to that for CLDR and there will be a therapeutic benefit if the overall TRT dose rate is kept fairly low.

For TRT, however, the associated LQ formulation must take account of the biological uptake and clearance kinetics in the targeted tumor or organ. This is because, with TRT, the critical dose-limiting tissues (e.g., the kidneys) may be anatomically distant from the tumor(s) to be treated and will likely be subjected to a quite different dose-time profile than the tumor. Thus, although the radiobiological processes which govern XTB, CLDR, and TRT are essentially identical, the separation of the tumor and doselimiting target organs makes quantitative analysis of TRT rather more difficult.

\section{EQUATIONS AND NUMERICAL EXAMPLES}

\section{Biologically Effective Doses (BEDs)}

Biologically effective doses (BEDs) allow for the quantitative assessment of the biological effects associated with different patterns of radiation delivery. The two principal radiobiological parameters required in such assessments are the tissue $\alpha / \beta$ ratio and the sublethal damage recovery constant $(\mu)$, both of which have been described elsewhere. ${ }^{1,3,4}$

In summary, $\alpha / \beta$ ratios provide a quantitative indication of the sensitivity of a given tumor or organ to changes in fractionation or dose rate. The $\alpha-\beta$ value (in units of Gy) is generally higher for tumors (typical range, 5-25 Gy) than for late-responding normal tissues (typical range, $2-5 \mathrm{~Gy}$ ). ${ }^{6}$ Damage-recovery constants are inversely related to the sublethal damage repair half-times, i.e., to the rate at which single-strand DNA breaks repair. The repair times are typically in the range of 0.5-3 hour and this is why, in external beam radiotherapy (where fractions are approximately 24 hour apart), the sublethal damage remaining after each fraction is usually fully repaired by the time of delivery of the next fraction. In CLDR or targeted treatments, the repair process takes place during the period of radiation delivery and is, therefore, in competition with the induction of further damage caused by the ongoing irradiation.

It should be noted that $\alpha / \beta$ values and, possibly, $\mu$ values also, are tissue-specific. Therefore, two tissue types, each receiving the same tem- poral pattern of radiation delivery, may well be associated with different BEDs.

BEDs are calculated by multiplying the total physical dose (TD) by a relative effectiveness (RE) factor, which takes account of the radiobiological parameters and also the type of radiation delivery (acute, protracted, diminishing doserate, and so forth), i.e.:

$$
\mathrm{BED}=\mathrm{TD} \times \mathrm{RE}
$$

For fractionated external beam therapy: ${ }^{2}$

$$
B E D=N d\left[1+\frac{d}{(\alpha / \beta)}\right]
$$

where $N$ is the number of fractions and $d$ the dose per fraction.

For continuous therapy at the low-dose rate: ${ }^{3,4}$

$$
B E D=R T\left[1+\frac{2 R}{\mu(\alpha / \beta)}\right]
$$

where $R$ is the dose-rate and $T$ the treatment time. It should be noted that Equation 2 is the simplified form of a more complex expression. ${ }^{3,4}$

For continuous therapy with an exponentially decreasing dose rate: ${ }^{3}$

$$
B E D=\frac{R_{o}}{\lambda}\left[1+\frac{R_{o}}{(\mu+\lambda)(\alpha / \beta)}\right]
$$

where $R_{o}$ is the initial dose-rate, and $\lambda$ is the overall rate constant describing the loss of activity (assumed to be exponential) from the organ in question, being the sum of the radioactive-decay constant and the organ-clearance constant. (It should be noted that the temporal pattern of activity uptake and clearance in targeted radiotherapy is complex and is not particularly well approximated by a monoexponential function, as is assumed in deriving Equation 3. ${ }^{7}$

Equations 1-3 may be used when there is no significant tissue repopulation during the course of the radiation delivery and are, therefore, appropriate for assessing late-responding normal tissues and slow-growing tumors. For acute-responding normal tissues and fast-growing tumors, both of which may exhibit cellular repopulation during treatment, more complex formulations are necessary as an extra dose is required to offset the repopulation effect. 8,9

As an example of the usefulness of BEDs, consider the following problem. It is desired to target a slow-growing tumor in order to deliver a biological dose which is equivalent to a course of external beam radiotherapy delivering $50 \mathrm{~Gy}$ 
in 25 fractions. Assuming that $\alpha / \beta$ for the tumour is $10 \mathrm{~Gy}$, the external beam BED is, from Equation 1:

$$
B E D=50 \times\left[1+\frac{2}{10}\right]=60 G y_{10}
$$

(The suffix after the $G y$ abbreviation indicates that values of $\alpha / \beta$ used in the calculation and serve as a reminder that this is a measure of biological, as opposed to physical, dose.) By equating Equation 3 to this desired BED value, we may identify monoexponentially decreasing patterns of radiation delivery, which will produce the same degree of tumor control as is being achieved by the external beam treatment.

$$
\frac{R_{o}}{\lambda}\left[1+\frac{R_{o}}{(\mu+\lambda)(\alpha / \beta)}\right]=60
$$

If, for example, it is known that the effective clearance half-life from the tumor is 12 hour, then $\lambda=0.693 / 12=0.0578$ hour $^{-1}$ and the initial dose rate may be computed from Equation 4. Assuming the half-life of repair for the tumor is 1 hour, then $\mu=0.603 / 1=0.693$ hour $^{-1}$ and:

$$
\frac{R_{o}}{0.0578}\left[1+\frac{R_{o}}{(0.693+0.0578) \times 10}\right]=60
$$

Leading to $R_{O}=2.581 \mathrm{~Gy} \mathrm{hour}^{-1}$, i.e., the total physical dose required from the targeted treatment would be $2.581 / \lambda=2.581 / 0.0578=44.7$ Gy.

If the clearance half-life from the tumor is significantly larger than the 12 hours assumed in this study, then a higher physical dose will be required for the same tumor effect, and vice versa. When the effective clearance rate is very slow, then the treatment will be delivered over a long time period and, as indicated earlier, in cases where there is significant tumor repopulation, yet more dose may be required to combat this. Such considerations are outside the scope of this paper but are discussed elsewhere. ${ }^{8}$

\section{ALLOWANCE FOR HIGH-LET (LINEAR ENERGY TRANSFER) RADIONUCLIDES}

The radiations used in conventional external beam treatments are mostly high-energy X-rays produced by linear accelerators. These have a low ionization density along the trajectories of the electrons liberated in the irradiated tissues and are hence classified as being low-LET (Linear Energy Transfer) radiations. Some of the radionu- clides used in targeted therapy exhibit high-LET characteristics and create a denser pattern of ionization. Thus, relative to the low-LET radiations used in conventional therapy, more biological damage is created for each unit of dose delivered. This enhancement is quantified in terms of relative biological effectiveness (RBE), the experimentally determined ratio of doses (low-LET versus high-LET) required to produce a given degree of cell kill, with the X-rays (by convention) being assigned an RBE of unity.

Incorporation of the RBE factor may be important for targeted radionuclide therapy as some of the nuclides could be associated with radiation emissions possessing RBEs significantly greater than unity. ${ }^{9,10}$ Allowance for RBE effects is easily made within the model because $\mathrm{RBE}$ maximizes to $\mathrm{RBE}_{\max }$ at low-dose rate (or low dose per fraction) and in Equations (1-3) it is necessary only to insert this parameter in place of the unity factor inside each of the square brackets. Equation 3, for instance, becomes:

$$
B E D=\frac{R_{o}}{\lambda}\left[R B E_{\max }+\frac{R_{o}}{(\mu+\lambda)(\alpha / \beta)}\right]
$$

It is always necessary to consider the possible existence of an enhanced RBE when using novel radionuclides. Returning to the previous example, if the radionuclide in question were known to be associated with an $\mathrm{RBE}_{\max }$ value of 1.6, then the Equation to be solved now becomes:

$$
\begin{aligned}
& \frac{R_{O}}{0.0578} \\
& \quad\left[1.6+\frac{R_{O}}{(0.693)+0.0578) \times 10}\right]=60
\end{aligned}
$$

that is to say $R_{o}=1.875 \mathrm{Gyh}^{-1}$, and the total dose now required is $1.875 / 0.0578=32.44 \mathrm{~Gy}$.

$\mathrm{RBE}$ effects are capable of exerting major influence on the radiobiological consequences of many forms of radiotherapy ${ }^{11-13}$ and, for targeted therapy, should always be considered, especially in relation to the dose-limiting normal structures. The interest in the use of alpha-emitters for TRT is a case in point where consideration of the RBE influence is vital (REFS).

\section{SUMMARY AND CONCLUSION}

In radiobiological terms, continuous low-doserate irradiation emulates the effects of external 
beam treatments delivered with many small fractions and is, therefore, a particularly effective type of radiotherapy. When tumors and critical normal tissues are each exposed to identical dose rates the use of a low-dose rate ensures that the differential sparing effect is likely to be beneficial and the LQ model provides a rational explanation of why this should be. TRT is a form of continuous low-dose-rate therapy for which the dose-rate is continually varying but, if the temporal pattern of dose-rate variation is known then, in principle, the LQ formulation may also be used to quantify TRT therapy.

As conventional radiobiological principles suggest that good normal tissue sparing is likely to result from using lower dose rates then, for TRT, this would suggest the use of nuclides with longer half-lives. However, with TRT, the radiobiological considerations cannot be considered in isolation from molecular vectoring efficiency and the biological environment. Other factors, such as the LET of the radionuclide emissions and tumor repopulation, may also be very relevant and should not be overlooked.

The BED concept is used widely in conventional radiotherapy and may also be used to quantify various forms of targeted radiotherapy. General consideration of the principal radiobiological issues is, therefore, possible with the LQ model, which can highlight the likely interactions between physical and biological factors. ${ }^{14}$ The principal caveats associated with modeling-based assessments of TRT are: (1) lack of knowledge of some radiobiological parameters, (2) difficulties in incorporating exact temporal uptake/clearance profiles into the model, and (3) limitations in the LQ model itself; it is useful but, of necessity, simplistic.

\section{ACKNOWLEDGMENT}

Elena Antoniou is thanked for her advice and practical help during the preparation of the conference presentation on which this paper is based.

\section{REFERENCES}

1. Dale RG. Use of the linear-quadratic radiobiological model for quantifying kidney response in targeted radiotherapy. Cancer Biother Radiopharm 2004;19:363.

2. Barendsen GW. Dose fractionation, dose rate, and isoeffect relationships for normal tissue responses. Int $J$ Radiat Oncol Biol Phys 1982;8:1981.

3. Dale RG. The application of the linear-quadratic doseeffect Equation uation to fractionated and protracted radiotherapy. Br J Radiol 1985;58:515.

4. Thames HD. An "incomplete-repair" model for survival after fractionated and continuous irradiations. Int $\mathrm{J} \mathrm{Ra}$ diat Biol 1985;47:319.

5. Fowler JF. The linear-quadratic model and progress in fractonation. Br J Radiol 1989;62:679.

6. Joiner MC, van der Kogel AJ. Basic Clinical Radiobiology, 3rd edition. Steel GG, ed. London: Arnold, 2002:120.

7. Howell RW, Goddu SM, Rao DV. Application of the linear-quadratic model to radioimmunotherapy: Further support for the advantage of longer-lived radionuclides. J Nucl Med 1994;35:1861.

8. Dale RG. Dose-rate effects in targeted radiotherapy. Phys Med Biol 1996;41:1871.

9. Antipas V, Dale RG, Coles IP. A theoretical investigation into the role of tumor radiosensitivity, clonogen repopulation, tumor shrinkage and radionuclide RBE in permanent brachytherapy implants of ${ }^{125} \mathrm{I}$ and ${ }^{103} \mathrm{Pd}$. Phys Med Biol 2001;46:2557.

10. Vandenbulcke $\mathrm{K}$, De Vos F, Offner F, et al. In vitro evaluation of ${ }^{213} \mathrm{Bi}$-rituximab versus external gamma irradiaiton for the treatment of B-CLL patients: Relative biological efficacy with respect to apoptosis induction and chromosomal damage. Eur J Nuc Med Mol Imaging 2003;30:1357.

11. Vrano M, Verhey LJ, Goitein M, et al. Relative biological effectiveness of modulated proton beams in various murine tumours. Int J Radiat Oncol Biol Physics 1984;10:509.

12. Gerweck LE, Kozin SV. Relative biological effectiveness of proton beams in clinical therapy. Radiother Oncol 1999;50:135.

13. Paganetti H, Niemierko A, Ancukiewicz M, et al. Relative biological effectiveness (RBE) values for proton beam therapy. Int J Radiat Oncol Biol Physics 2002;53:407.

14. O'Donoghue JA. Relevance of external beam dose-response relationships to kidney toxicity associated with radionuclide therapy. Cancer Biother Radiopharm 2004; $19: 378$. 